\title{
The Influence of Edges as Salient Features in Haptic Shape Perception of 3D Objects
}

\author{
Virjanand Panday* Wouter M. Bergmann Tiest $\quad$ Astrid M. L. Kappers
}

Helmholtz Institute, Utrecht University, The Netherlands

\begin{abstract}
Salient features help identify objects. However, they can also influence subsequent comparison between objects. In this study, we investigate the influence of edges on perception of the shape of 3D objects. We tested two conditions in which subjects were asked to indicate the orientation of a block by exploring it either statically without touching the edges or freely. When edges were excluded, the threshold to determine the orientation of a block was an aspect ratio of 1.030. When the edges were explored, the threshold was 1.045. We conclude that edges disrupt the precision at which $3 \mathrm{D}$ objects can be perceived.
\end{abstract}

Index Terms: J.4 [Social and Behavioral Sciences]: Psychology-Haptic perception

\section{INTRODUCTION}

The world around us is filled with 3D objects. In our normal daily life we pick up and use many of these objects, usually without thinking about it and often without even looking at them. At other times, we are unable to use our eyes to find objects, for instance in the dark, in bags or pockets. We identify and distinguish between objects by using the physical properties of these objects. One property we use to identify or distinguish objects is shape. A very salient feature in shape is an edge. If an object with edges has to be found between objects without edges, this can be done very easily [8]. When building haptic devices or virtual environments, edges should therefore be an important feature. However, is the presence of edges always advantageous?

In identifying 3D objects, humans use different exploration strategies to distinguish different features of the object. Lederman and Klatzky [6] found that when subjects were asked to explore the shape of objects they made enclosing and contour following movements. This indicates that different features of a shape are being explored by different exploratory procedures. It is therefore interesting to identify which features are being explored and what their relative contributions are.

In 3D shape perception, two important aspects are curvature and edges. In studies concerned with 3D shape perception, the focus on either curvature or edges can be seen from the stimuli used. For example, in Lakatos and Marks [5], polyhedrons were used to investigate the importance of local versus global features in object perception. In contrast, Norman et al. [7] used shapes of bell peppers to compare 3D shapes with each other both haptically and visually. Other studies used stimuli that directly compared the influence of curvature and edges. Roland and Mortensen [9] used both ellipsoids and parallelepipeds to show that shapes with differences in curvature yielded higher performance in discriminating the more oblong object.

Van der Horst and Kappers [10] found that when humans are asked to distinguish a cylinder with an elliptical cross-section from

*e-mail: V.Panday@uu.nl

IEEE World Haptics Conference 2011

21-24 June, Istanbul, Turkey

978-1-4577-0297-6/11/\$26.00 @2011 IEEE a cylinder with a circular cross-section, they can distinguish a four times lower difference in aspect ratio than when distinguishing between a block with a rectangular cross-section and a block with a square cross-section. They concluded from this that curvature information can be used in a reliable manner.

On the other hand, it has been shown by Plaisier et al. [8] that edges and vertices are the most salient features in a haptic search task. In this search task, a target of one shape had to be detected among distractor items of a different shape. The shapes consisted of cubes, spheres, tetrahedrons, cylinders and ellipsoids. They compared the search slopes and concluded that edges and vertices were the dominant parameters for search efficiency.

From these studies it follows that on the one hand humans perform better in detection and discrimination tasks on objects that have primarily curvature properties than on objects that have strong edges $[10,9]$. On the other hand, it has been shown that edges are strong features that are very salient in object detection [8]. Salient features can therefore be concluded to be good for recognizing and detecting objects. In other tasks, however, where the salient features do not directly contribute, they may disrupt the accuracy with which the task is performed.

For example, in Van der Horst and Kappers [10] it has been shown that the performance on blocks is four times as inaccurate as the performance on ellipsoids. They conclude from this that the additional curvature information in the ellipses is used in a reliable manner. Another explanation could be that the edges play a crucial role. Edges are salient features, as indicated by Plaisier et al. [8], that are good for recognizing and detecting objects. However, in the perception of the aspect ratio, where the presence of edges does not directly contribute, they may disrupt the accuracy with which this task is performed. Whether the salient nature of the edges disrupts the perception of the aspect ratio or whether the better performance in ellipsoids is caused by the additional curvature information, is a fundamental question.

In this study, we investigated whether the presence of strongly salient properties in an object causes a lower performance in discrimination tasks of humans on such objects. We investigated whether the presence of edges disrupts the perception of the aspect ratio in blocks. Subjects were asked to identify the orientation of a block with a rectangular cross-section in two conditions. In the first condition, they were instructed to grasp two opposing sides of the block statically while avoiding the edges and to repeat this for the other two opposing sides. In the second condition, they were asked to grasp the block and explore it freely. The aspect ratio of the cross-sections of the rectangles varied from almost square to more rectangular, so that a threshold could be determined.

One could expect that when subjects are asked to explore the block freely, they would be able to determine the orientation of the block correctly for smaller aspect ratios, because they can access more information, such as for example the length of the sides, which could also be perceived by sliding the fingers along the total length of the side. On the other hand, one could expect that by avoiding the edges in the exploration of the block, the disturbing influence of the edges is eliminated, leading to lower thresholds. The following experiment will investigate these conflicting possibilities. 


\section{Methods}

\subsection{Subjects}

Eleven paid subjects (mean age $21 \pm 3$ years, 6 male) participated in the experiment. The result of one of these subjects was not included, since this subject employed a strategy which was not based on grasping. One subject (BK) was strongly left-handed, one subject (YB) was moderately right-handed all other subjects were strongly right-handed according to Coren's test [2]. All subjects were naive as to the purpose of the experiment and gave their informed consent. None of the subjects reported any known hand deficits.

\subsection{Stimuli}

Blocks with a rectangular cross-section were used as stimuli. The blocks were made of a compound of polyurethane foam and artificial resin (Cibatool BM 5460) and manufactured on a computer controlled milling machine. The stimuli were similar to the stimuli used by Van der Horst and Kappers [10], but of slightly different dimensions. The height was $150 \mathrm{~mm}$. The areas of the cross-sections of the rectangles were equal $\left(2450 \mathrm{~mm}^{2}\right)$. The stimuli are defined in terms of the aspect ratio of the horizontal cross-section $(\alpha)$, which is defined as the quotient of the longest axis $(a)$ and the shortest axis (b) as depicted in figure 1 . For the condition where the subjects explored the stimuli statically while avoiding the edges, test stimuli with aspect ratios of $1.004,1.006,1.010,1.016,1.020,1.04,1.06$ and 1.08 were used. For the condition where the subjects explored the stimuli freely, test stimuli with aspect ratios of 1.006, 1.010, $1.020,1.04,1.06,1.08,1.1$ and 1.13 were used. The ranges of the test stimuli were based on pilot experiments. An image of the stimuli used can be seen in figure 2 .

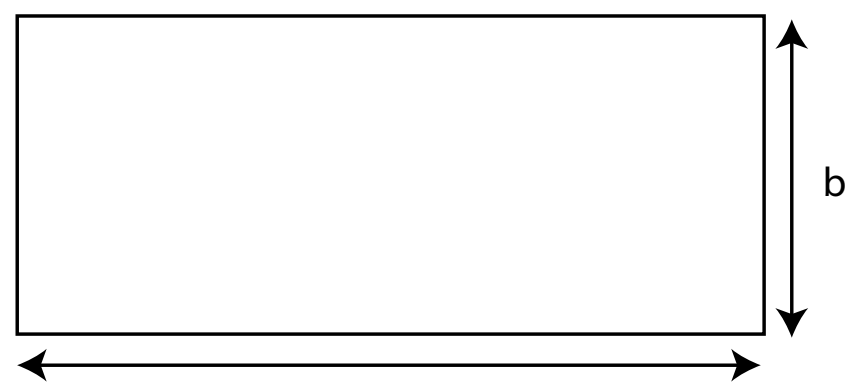

a

Figure 1: Schematic illustration of the rectangular cross-section of the stimuli with long axis $a$ and short axis $b$.

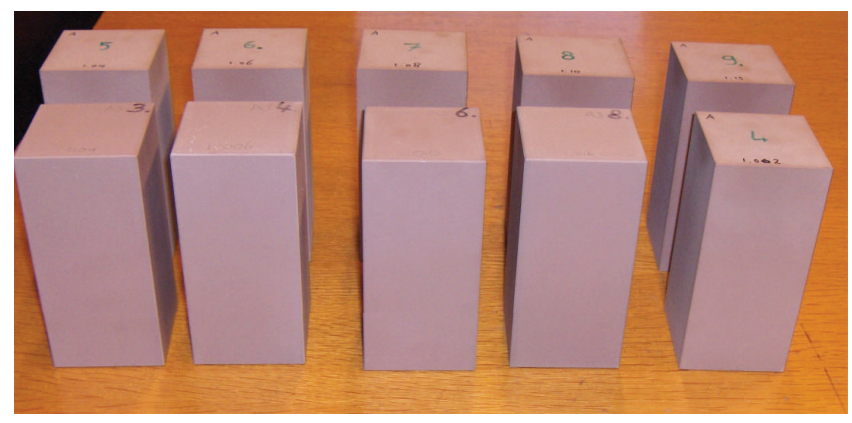

Figure 2: Set of stimuli used.

\subsection{Procedure}

The subjects were seated behind a curtain which prevented them from seeing the experimenter and the stimuli. The stimulus was placed on the table behind the curtain, in such a way that the subject could comfortably reach around the stimulus. The subjects had to indicate from a drawing, shown in figure 3 , in which orientation they felt the block was standing behind the curtain. This drawing was located in front of the curtain.

On the drawing two blocks were depicted. For half the subjects the left block had the longest axis from the bottom left to the top right, while the right block was rotated $90^{\circ}$. For the other half this was the other way around. The subjects were asked to indicate whether the block they felt was oriented in the same direction as either the left or the right block on the drawing.

During a trial the subjects put their dominant hand under the curtain and felt the stimulus in one of two ways. During exploration the experimenter held the block in position by holding it at the top. In the first condition the subjects were asked to grab two opposing sides of the block between their thumb and the other four fingers, making sure not to come into contact with the edges. They then let go and turned their hand around the stimulus without touching it, to grab the other two opposing sides in a similar manner. An image of the hand positions in the first condition is given in figure 4 . In the second condition they were asked to grab the block and were left free to explore the block as they wished.
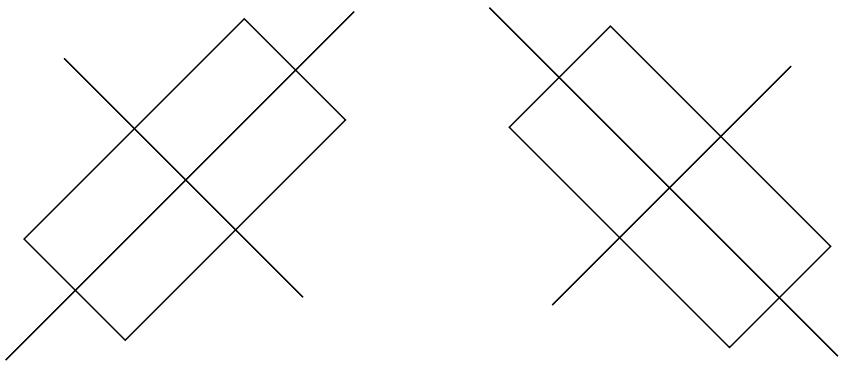

Figure 3: Visual depiction of the two orientations, from which the subjects had to choose.

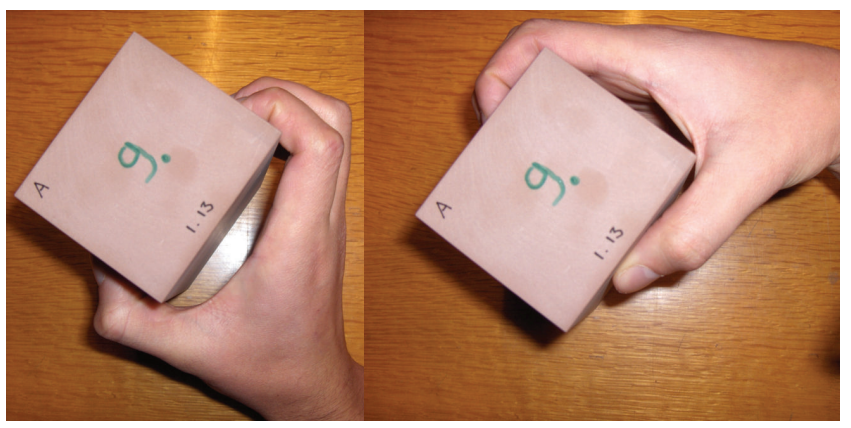

Figure 4: Photograph of the procedure in the first condition.

Each condition consisted of 80 trials. Each stimulus was presented five times in both orientations, giving ten trials per stimulus. The stimuli were presented successively in a random sequence. The order in which the conditions were measured was counterbalanced across subjects. Each session with two conditions took 40-80 min.

\subsection{Analysis}

The fraction of correct responses was plotted against the relative aspect ratio $(\alpha-1)$ on a logarithmic scale. The logarithmic scale 
is used to show that for relative aspect ratios close to zero, the percentage correct is at chance level $(50 \%)$. This use of a logarithmic scale is justified because it is reasonable to assume that the detection threshold for relative aspect ratio shows a Weber-like behavior. This means that the perceived size of a constant physical difference decreases with increasing aspect ratio. On a logarithmic scale, equal perceived differences would then be equidistant. To the fraction of correct judgments, a psychometric function of the form

$$
g(x)=\frac{1}{4} \operatorname{erf}\left(\frac{{ }^{10} \log \left(\frac{x}{p}\right)}{\sqrt{2} \sigma}\right)+\frac{3}{4}
$$

was fitted. Here, erf is the cumulative Gaussian distribution, $p$ is the relative aspect ratio of the $75 \%$ point (the point halfway between chance level and $100 \%$ correct) and $\sigma$ is a parameter that describes the steepness of the curve. This function, described in Bergmann Tiest and Kappers [1], was chosen to enable an analysis that assumes that performance is at chance level when the relative aspect ratio reaches zero. Furthermore this function is antisymmetric on a logarithmic scale. The relative aspect ratio where the function equals 0.75 was taken as the detection threshold. An example for subject YB of the two conditions is shown in figure 5.

\section{Results}

Figure 6 shows the mean detection thresholds for ten subjects in two conditions. The average threshold for the first condition, where subjects were instructed to grasp the stimuli statically while avoiding the edges, was a relative aspect ratio of 0.030 . The threshold for the second condition where subjects were free to explore the stimuli, was a relative aspect ratio of 0.045 . A two-tailed paired t-test showed that condition had a significant effect $(t=2.7, p=0.024)$. On average subjects were able to perceive the orientation of blocks with lower aspect ratios for the first condition compared to the second condition.

\section{Discussion}

These results show that when subjects are allowed to feel the edges, the performance decreases compared to when they are instructed to avoid the edges. The mean threshold for the condition in which the edges were avoided is significantly lower. This indicates that edges have a disrupting influence in perception tasks not involving the edges. We can conclude that free exploration of a block, even though there is more information available to the subject, does not lead to lower thresholds. Instead, avoiding the edges in the exploration of the block, thus eliminating the disturbing influence of the edges, does lead to lower thresholds.

Van der Horst and Kappers [10] showed that subjects have four times lower thresholds for cylinders than for blocks. The difference between the conditions could be explained by the additional presence of curvature information in the cylindrical stimuli in their experiments. An alternative explanation is that the salient edges in their rectangular blocks had a disruptive effect on performance. In our experiment we investigated this possibility. By comparing two conditions directly without additional curvature information, we quantitatively identified the influence of edges on discrimination tasks: Edges disrupted perception by increasing the threshold of discrimination with $50 \%$.

The experiment done by Van der Horst and Kappers [10] gives a much higher threshold for the condition where blocks were explored freely than our condition in which the blocks were explored freely. This is probably due to the different task that the subjects were asked to perform. In Van der Horst and Kappers the subjects had to choose between two stimuli that were offered one after the other. They had to indicate which one was the block with a square cross-section. In our experiment, however, the subjects had only

\section{Opposing fingers, edges avoided}

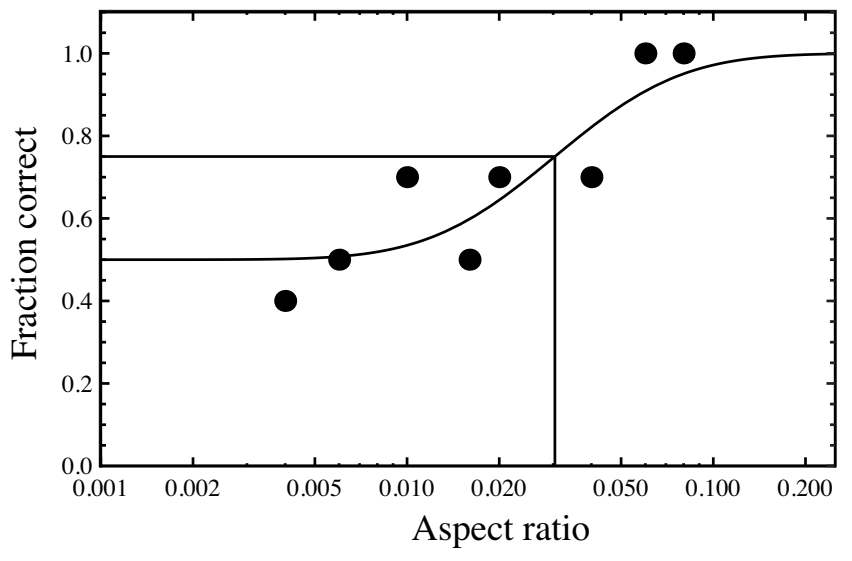

Free exploration

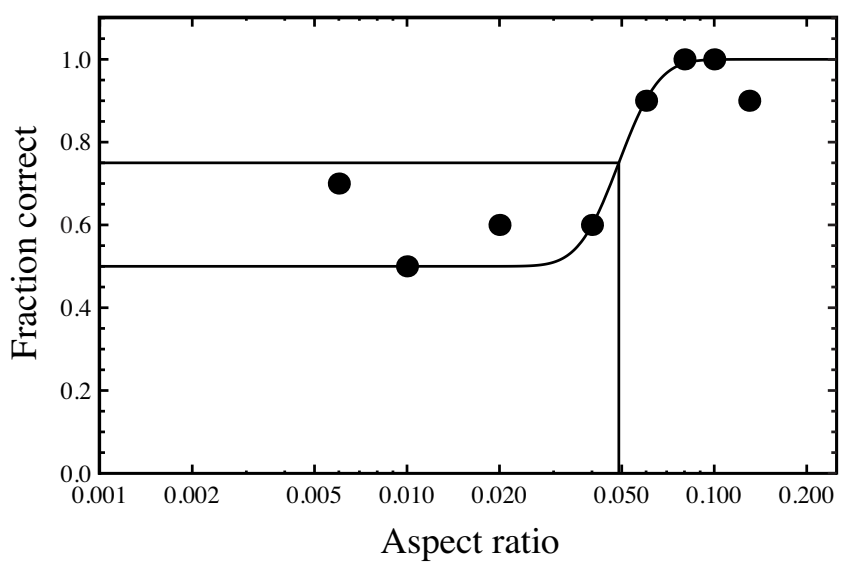

Figure 5: Examples of psychometric curves for condition 1 (opposing fingers, edges avoided) and condition 2 (free exploration) of subject $Y B$. The response is plotted against the relative aspect ratio, which is defined as the aspect ratio minus 1 . Note that a logarithmic scale is used. A psychometric function was fitted to the data. The detection threshold is defined as the relative aspect ratio value for which the psychometric function equals 0.75 .

one block to explore. They had to indicate in what orientation they felt the block was positioned by comparing it to a visual depiction of the two possible orientations. In the experiment of Van der Horst and Kappers more actions had to be performed and the comparison was done in memory. Furthermore, the orientations of the blocks were randomized. These conditions make performing the task more complicated and could explain the higher thresholds.

The main source of information that can be used to perform the task in our experiment from the stimuli is the difference in length of the two perpendicular axes. Both Durlach et al. [3] and Gepshtein and Banks [4] reported thresholds for length perception by grasping two parallel plates between the index finger and the thumb. Durlach et al. employed a physical device with rectangular pads, which had to be grasped between the thumb and the forefinger. Subjects were presented with a short $(L)$ or a long $(L+\Delta L)$ stimulus and were asked to judge which of the two had been presented. Three reference lengths were tested with two increments for each reference length. They found that on a reference length of $40 \mathrm{~mm}$ the Just Noticeable Difference (JND) is $1.9 \mathrm{~mm}$. This can be expressed in 


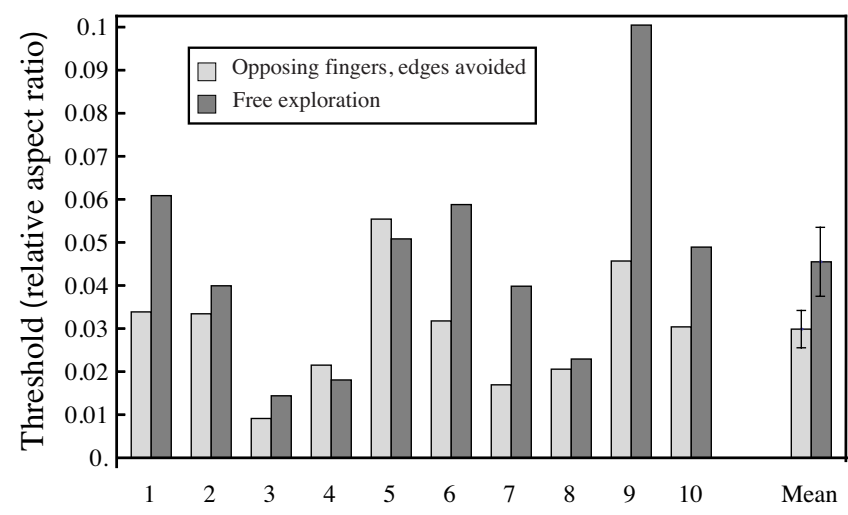

Figure 6: Detection threshold results of ten subjects in two conditions (opposing fingers, edges avoided and free exploration) and the average result. The error bars on the average result represent standard errors.

terms of our stimuli, by using equation 2 .

$$
\alpha-1=\frac{L+\Delta L}{L}-1
$$

This means that a threshold for the relative aspect ratio based on this length discrimination JND would be 0.048 . This result is higher than our results of both the first and second condition. One would expect the result of Durlach et al. to be nearer to the result of the first condition, because in this condition two lengths are grasped between the thumb and the four other fingers, without touching the edges. Furthermore in Durlach et al. there are no edges to disturb the perception. A possible reason for the higher threshold of Durlach et al. is that in their experiment two subjects performed the task with their dominant hand and two subjects with their nondominant hand. This might explain the higher average threshold. Moreover, the variation between subjects is quite large in this kind of experiments, as evidenced by the spread in our own results. With only four subjects, Durlach et al. are even more susceptible to such fluctuations, making it likely that the thresholds do not coincide.

Gepshtein and Banks [4] represented the plates virtually using PHANToM force-feedback devices. Subjects were presented six test stimuli and had to compare these to a reference stimulus of 50 $\mathrm{mm}$. They were asked to indicate which of the two stimuli contained the greater intersurface distance. They found a JND of 5 $\mathrm{mm}$ on a $50 \mathrm{~mm}$ reference stimulus. This JND was determined at 1 standard deviation, which corresponds to $84 \%$ correct. To convert this into $75 \%$ one must apply a conversion factor of 0.67 . Using equation 2 and the conversion factor makes it possible to express the JND of Gepshtein and Banks into a relative aspect ratio of 0.07 , which can be compared to our results. This result is higher than our results of both conditions. One would expect the result of Gepshtein and Banks to be around the result of the first condition, because in this condition two lengths are grasped between the thumb and the four other fingers, without touching the edges. A possible explanation could be that the accuracy with which humans can perform perception tasks is negatively affected by haptic devices.

\section{Conclusion}

This experiment shows that edges as salient features interfere with the accuracy of the perception of 3D objects. Salient features are important in the identification of objects and stand out. We show that this might have a negative consequence for the subsequent perception of less salient features of the object. In selection tasks where the objects are very similar, the presence of salient features probably hinders the performance of humans. We can therefore conclude that the presence of edges is not always an advantage.

\section{ACKNOWLEDGEMENTS}

This work has been partially supported by the European Commission with the Collaborative Project no. 248587, "THE Hand Embodied", within the FP7-ICT-2009-4-2-1 program "Cognitive Systems and Robotics"

\section{References}

[1] W. M. Bergmann Tiest and A. M. L. Kappers. An antisymmetric psychometric function on a log scale. Perception, in press.

[2] S. Coren. Measurement of handedness via self-report: the relationship between brief and extended inventories. Perceptual and motor skills, 76(3 Pt 1):1035-1042, 1993.

[3] N. I. Durlach, L. A. Delhorne, A. Wong, W. Y. Ko, W. M. Rabinowitz, and J. Hollerbach. Manual discrimination and identification of length by the finger-span method. Perception \& Psychophysics, 46(1):29-38, 1989.

[4] S. Gepshtein and M. S. Banks. Viewing geometry determines how vision and haptics combine in size perception. Current Biology, 13(6):483-488, 2003.

[5] S. Lakatos and L. E. Marks. Haptic form perception: Relative salience of local and global features. Perception \& Psychophysics, 61(5):895-908, 1999.

[6] S. J. Lederman and R. L. Klatzky. Hand movements: A window into haptic object recognition. Cognitive psychology, 19 (3):342-368, 1987.

[7] J. F. Norman, H. F. Norman, A. M. Clayton, J. Lianekhammy, and G. Zielke. The visual and haptic perception of natural object shape. Perception \& Psychophysics, 66(2):342-351, 2004.

[8] M. A. Plaisier, W. M. Bergmann Tiest, and A. M. L. Kappers. Salient features in 3-D haptic shape perception. Attention, Perception, \& Psychophysics, 71(2):421-430, 2009.

[9] P. E. Roland and E. Mortensen. Somatosensory detection of microgeometry, macrogeometry and kinesthesia in man. Brain Research Reviews, 12(1):1-42, 1987.

[10] B. J. Van der Horst and A. M. L. Kappers. Using curvature information in haptic shape perception of 3D objects. Experimental Brain Research, 190(3):361-367, 2008. 